\title{
NONNEGATIVE SOLUTIONS TO SOME SINGULAR SEMILINEAR ELLIPTIC PROBLEMS
}

\author{
TOMÁS GODOY*, ALFREDO GUERIN
}

FAMAF, Universidad Nacional de Córdoba, Ciudad Universitaria, Córdoba 5000, Argentina

\begin{abstract}
We prove the existence of a nonnegative weak solution $0 \not \equiv u \in H_{0}^{1}(\Omega)$ to the singular semilinear elliptic problem $-\Delta u=\chi_{\{u>0\}} a u^{-\alpha}+f(., u)$ in $\Omega, u=0$ on $\partial \Omega$, where $\Omega$ is a bounded domain in $\mathbb{R}^{n}, 0<\alpha<3$, $a \in L^{\infty}(\Omega), 0 \not \equiv a \geq 0$, and $f: \Omega \times[0, \infty) \rightarrow \mathbb{R}$ is a Carathéodory function which satisfies some suitable hypothesis. We also obtain results about the problem with a parameter $-\Delta u=\chi_{\{u>0\}} a u^{-\alpha}+\lambda f(., u)$ in $\Omega, u \geq 0$ in $\Omega, u=0$ on $\partial \Omega$.
\end{abstract}

Keywords. Singular elliptic problem; Variational technique; Nonnegative solution; Bifurcation problem.

2010 Mathematics Subject Classification. 35J20, 35J60, 35J75.

\section{Introduction}

Let us consider the singular semilinear elliptic problem:

$$
\left\{\begin{array}{l}
-\Delta u=\chi_{\{u>0\}} a u^{-\alpha}+f(x, u) \text { in } \Omega \\
u=0 \text { on } \partial \Omega \\
u \geq 0 \text { in } \Omega, u \neq 0 \text { in } \Omega
\end{array}\right.
$$

${ }^{*}$ Corresponding author.

E-mail addresses: tomasgodo@gmail.com (T. Godoy), guerin.alfredojose@gmail.com (A. Guerin).

Received July 30, 2016; Accepted December 5, 2016. 
and the related problem with a parameter $\lambda$ :

$$
\left\{\begin{array}{l}
-\Delta u=\chi_{\{u>0\}} a u^{-\alpha}+\lambda f(x, u) \text { in } \Omega, \\
u=0 \text { on } \partial \Omega, \\
u \geq 0 \text { in } \Omega, u \neq 0 \text { in } \Omega .
\end{array}\right.
$$

where $\Omega$ is a bounded domain in $\mathbb{R}^{n}$ with $C^{1,1}$ boundary, $0<\alpha<3, \lambda \in \mathbb{R}, a, f$ are functions defined on $\Omega$ and $\Omega \times[0, \infty)$ respectively; and where $\chi_{\{u>0\}} a u^{-\alpha}$ stands for the function defined by $\chi_{\{u>0\}} a u^{-\alpha}(x):=a(x) u(x)^{-\alpha}$ if $u(x) \neq 0$, and $\chi_{\{u>0\}} a u^{-\alpha}(x):=0$ if $u(x)=0$.

These problems have received considerable interest in the literature and appear in applications to chemical catalysts process, non-Newtonian fluids, and in models for the temperature of electrical conductors (see e.g., [6], [4], [12], [15] and the references therein). The existence of positive solutions (i.e. such that $u(x)>0$ for all $x \in \Omega$ ) to problem (1.1) was proved, for the case $f=0$, and under various assumptions on $a$, in [15], [12], [7], [21], [10] and [3]. Existence theorems for positive classical solutions to problem (1.2) were obtained by Shi and Yao in [24], when $\Omega$ and $a$ are regular enough, with $a$ non necessarily nonnegative, $f(x, s)=s^{p}$ and $0<\alpha, p<1$. The free boundary singular elliptic bifurcation problem $-\Delta u=\chi_{\{u>0\}}\left(-u^{-\alpha}+\lambda f(., u)\right)$ in $\Omega, u=0$ on $\partial \Omega, u \geq 0$ in $\Omega, u \not \equiv 0$ (that is: $|\{x \in \Omega: u(x)>0\}|>0$ ) was studied by Dávila and Montenegro in [9], under the assumptions that $0<\alpha<1, \lambda>0, f: \Omega \times \mathbb{R} \rightarrow \mathbb{R}$ is a nonnegative Carathéodory function, $f(x, s)$ is nondecreasing and concave in $s$, and $\lim _{s \rightarrow \infty} f(x, s)=0$ uniformly on $x \in \Omega$.

Bifurcation problems of the form $-\Delta u=g(x, u)+f(x, \lambda u)$ in $\Omega, u=0$ on $\partial \Omega, u>0$ in $\Omega$, were studied by Coclite and Palmieri [5]. It was proved there that, if $g(x, u)=a u^{-\alpha}, a \in$ $C^{1}(\bar{\Omega}), a>0$ in $\bar{\Omega}$, and $f \in C^{1}(\bar{\Omega} \times[0, \infty))$, then there exists $\lambda^{*}>0$ such that, for any $\lambda \in$ $\left[0, \lambda^{*}\right),(1.2)$ has a positive classical solution $u \in C^{2}(\Omega) \cap C(\bar{\Omega})$. Furthermore; for any $\lambda \geq 0$, a positive classical solution exists if, in addition, $\varlimsup_{s \rightarrow \infty} \frac{f(x, s)}{s} \leq 0$ uniformly on $x \in \bar{\Omega}$ (see [5], Theorem 1).

Multi-parameter singular bifurcation problems of the form $-\Delta u=g(u)+\lambda|\nabla u|^{p}+\mu f(., u)$ in $\Omega, u=0$ on $\partial \Omega, u>0$ in $\Omega$ were studied, by Ghergu and Rădulescu in [18]. Dupaigne, Ghergu and Rădulescu [14] obtained existence and nonexistence theorems for Lane-Emden-Fowler equations with convection and singular potential. Rădulescu [23] stated existence, nonexistence, and uniqueness theorems for blow-up boundary solutions of logistic equations, and for Lane-Emden-Fowler equations, with singular nonlinearities and 
subquadratic convection term. Existence and nonexistence results for positive solutions to the inequality $L u \geq K(x) u^{p}$ on the punctured ball $\Omega=B_{r}(0) \backslash\{0\}$ were obtained by Ghergu, Liskevich and Sobol [16] for second order linear elliptic operators $L$ without zero order term, and $K \in L_{\text {loc }}^{\infty}(\Omega)$ such that $0<e s s \inf K$. A Liouville comparison principle for entire weak solutions of quasilinear singular parabolic second-order partial differential inequalities was obtained in [20] by Kurta and existence and uniqueness results were obtained by Bougherara and Giacomoni [1] for mild solutions to singular initial value parabolic problems involving the p-Laplacian. Singularly perturbed elliptic problems on an annulus whose solutions concentrate in a circle were studied by Manna and Srikanth [22].

The following problem

$$
\left\{\begin{array}{l}
-\Delta u=a g(u)+\lambda f(u) \text { in } \Omega, \\
u=0 \text { on } \partial \Omega, \\
u>0 \text { in } \Omega .
\end{array}\right.
$$

was considered by Cîrstea, Ghergu and Rădulescu [8] under the following assumptions: $\Omega$ is a regular enough bounded domain in $\mathbb{R}^{n}, 0 \leq a \in C^{\beta}(\bar{\Omega}), 0<f \in C^{0, \beta}[0, \infty)$ for some $\beta \in(0,1), f$ is nondecreasing on $[0, \infty), f(s) / s$ is nonincreasing for $s>0, g$ is nonincreasing on $(0, \infty), \lim _{s \rightarrow 0^{+}} g(s)=+\infty$; and there exist $\alpha \in(0,1), \sigma_{0}>0$, and $c>0$, such that $g(s) \leq c s^{-\alpha}$ for $s \in\left(0, \sigma_{0}\right)$. Under these hypothesis, and defining $\mu:=\lim _{s \rightarrow \infty} f(s) / s$, $\lambda^{*}:=\lambda_{1} / \mu$ (where $\lambda_{1}$ stands for the first Dirichlet eigenvalue of $-\Delta$ in $\Omega$ ), and $\mathscr{E}:=\left\{u \in C^{2}(\Omega) \cap C^{1,1-\alpha}(\bar{\Omega}): \Delta u \in L^{1}(\Omega)\right\}$, the following results were proved:

([8], Theorem 1): If $\mu=0$ and $\min _{\bar{\Omega}} a>0$ (respectively $\min _{\bar{\Omega}} a=0$ ), then, for all $\lambda \in \mathbb{R}$ (resp. $\lambda \geq 0$ ), problem (1.3) has a unique solution $u_{\lambda} \in \mathscr{E}$, the map $\lambda \rightarrow u_{\lambda}$ is strictly increasing, and each $u_{\lambda}$ satisfies $c_{1} d_{\Omega} \leq u_{\lambda} \leq c_{2} d_{\Omega}$ for some positive constants $c_{1}$ and $c_{2}$, where $d_{\Omega}:=$ $\operatorname{dist}(., \partial \Omega)$

([8], Theorem 2): If $\mu>0$ and $\lambda \geq \lambda^{*}$, then (1.3) has no solutions in $\mathscr{E}$. Furthermore, if $\mu>0$ and $\min _{\bar{\Omega}} a>0$ (respectively $\min _{\bar{\Omega}} a=0$ ), then (1.3) has a unique solution $u_{\lambda} \in \mathscr{E}$ for any $\lambda<\lambda^{*}$ (resp. $0 \leq \lambda<\lambda^{*}$ ) and, again, the map $\lambda \rightarrow u_{\lambda}$ is strictly increasing; and each $u_{\lambda}$ satisfies $c_{1} d_{\Omega} \leq u_{\lambda} \leq c_{2} d_{\Omega}$ for some positive constants $c_{1}$ and $c_{2}$. Moreover, $\lim _{\lambda \rightarrow\left(\lambda^{*}\right)^{-}} u_{\lambda}=$ $+\infty$ uniformly on compact subsets of $\Omega$. 
Finally, let us mention that in [19], the authors proved the existence of nonnegative solutions for a restricted version of problem (1.1), namely when $H 1$ ) holds, $0<\alpha<1$, and $f(., u)=$ $-b u^{p}$, with $0<p<\frac{n+2}{n-2}$, and $0 \leq b \in L^{r}(\Omega)$ for suitable values of $r$.

Additional references, and a comprehensive treatment of the subject, can be found in [17], [23], see also [11].

The aim of this work is to prove, under suitable hypothesis on $a$ and $f$, existence results for nonnegative weak solutions to problems (1.1) and (1.2). By a weak solution we mean a solution in the sense of the following.

Definition 1.1. We say that $u: \Omega \rightarrow \mathbb{R}$ is a weak solution of problem (1.1) if $u \in H_{0}^{1}(\Omega), u \geq 0$, $\chi_{\{u>0\}} a u^{-\alpha} \varphi \in L^{1}(\Omega)$ and

$$
\int_{\Omega}\langle\nabla u, \nabla \varphi\rangle=\int_{\Omega} \chi_{\{u>0\}} a u^{-\alpha} \varphi+\int_{\Omega} f(x, u) \varphi .
$$

for all $\varphi$ in $H_{0}^{1}(\Omega) \cap L^{\infty}(\Omega)$.

For $b \in L^{\infty}(\Omega)$ such that $b^{+} \not \equiv 0$, we will write $\lambda_{1}(b)$ for the positive principal eigenvalue for $-\Delta$ on $\Omega$, with homogeneous Dirichlet boundary condition and weight function $b$. With this notation, our first result reads as follows.

Theorem 1.2. Let $\alpha \in(0,3)$ and assume the following conditions:

H1) $a \in L^{\infty}(\Omega), a \geq 0$, and $a \not \equiv 0$,

H2) $f: \Omega \times[0, \infty) \rightarrow \mathbb{R}$ is a Carathéodory function on $\Omega \times[0, \infty)$, i.e., $f(., s)$ is measurable for any $s \in[0, \infty)$, and $f(x,$.$) is continuous a.e. x \in \Omega$,

H3) $\sup _{0 \leq s \leq M}|f(., s)| \in L^{1}(\Omega)$ for any $M>0$,

H4) One of the two following conditions holds:

$\left.H 4^{\prime}\right) \sup _{s>0} \frac{f(., s)}{s} \leq b$ for some $b \in L^{\infty}(\Omega)$ such that $b^{+} \not \equiv 0$, and $\lambda_{1}(b)>m$ for some integer $m \geq \max \{2,1+\alpha\}$,

H4”) $f \in L^{\infty}(\Omega \times(0, \sigma))$ for all $\sigma>0$, and $\varlimsup_{\lim _{s \rightarrow \infty}} \frac{f(., s)}{s} \leq 0$ uniformly on $\Omega$, i.e., for any $\varepsilon>0$ there exists $s_{0}>0$ such that $\sup _{s \geq s_{0}} \frac{f(., s)}{s} \leq \varepsilon$, a.e. in $\Omega$,

H5) $f(., 0) \geq 0$.

Under these hypothesis, (1.1) has a weak solution $u$ (in the sense of Definition 1.1), that belongs to $H_{0}^{1}(\Omega) \cap L^{\infty}(\Omega)$; and satisfies:

i) $u>0$ a.e. in $\{a>0\}$. In particular, $\chi_{\{u>0\}} a u^{-\alpha} \not \equiv 0$ and, if $a>0$ a.e. in $\Omega$, then $u>0$ a.e. in $\Omega$. 
ii) If $f(., 0)>0$ a.e. in $\Omega$, then $u>0$ a.e. in $\Omega$.

Note that, if $f \geq 0$ in $\Omega \times[0, \infty)$ then, by the maximum principle, the solutions to problem (1.1) that satisfy $\chi_{\{u>0\}} a u^{-\alpha} \not \equiv 0$ are positive a.e. in $\Omega$. Example 3.7 in [19] shows that conditions like the ones stated above are needed in order to ensure the existence of a strictly positive weak solution.

Concerning problem (1.2) our results are the following.

Theorem 1.3. Let $\alpha \in(0,3)$, and assume that H1)-H3), H4”) and H5) hold. Then, for all $\lambda \geq 0,(1.2)$ has a weak solution $u_{\lambda}$ (in the sense of Definition 1.1); this solution $u_{\lambda}$ is in $H_{0}^{1}(\Omega) \cap L^{\infty}(\Omega)$, satisfies $\chi_{\{u>0\}} a u_{\lambda}^{-\alpha} \not \equiv 0$ and $u_{\lambda}>0$ a.e. in $\{a>0\}$. These results remain valid for any negative $\lambda$ if, in addition, $f(., 0)=0$ and $\lim _{s \rightarrow \infty} \frac{f(., s)}{s}=0$ uniformly on $\Omega$.

Moreover, for $\lambda \geq 0$, if $f(., 0)>0$ a.e. in $\Omega$, then $u_{\lambda}>0$ a.e. in $\Omega$.

Theorem 1.4. Let $\alpha \in(0,3)$; assume H1)-H3), H5), and that one of the two following conditions holds:

H6) $\operatorname{esssup}_{(x, s) \in \Omega \times(0, \infty)} \frac{f(x, s)}{s}<\infty$,

H7) $f \in L^{\infty}(\Omega \times(0, \sigma))$ for all $\sigma>0$.

Then there exists $\lambda^{*}>0$ such that, for any nonnegative $\lambda<\lambda^{*}$, (1.2) has a weak solution (in the sense of Definition 1.1) $u_{\lambda} \in H_{0}^{1}(\Omega) \cap L^{\infty}(\Omega)$ that satisfies $\chi_{\left\{u_{\lambda}>0\right\}}$ au $\lambda_{\lambda}^{-\alpha} \not \equiv 0$ and $u_{\lambda}>0$ a.e. in $\{a>0\}$.

Theorems 1.3 and 1.4, can be viewed as partial generalizations of the already mentioned existence results contained in [8]. Let us briefly compare those results with ours: On the one hand, our assumptions on $a$ and $f$ are weaker than those imposed in [8]: we allow that $|\{x \in \Omega: a(x)=0\}|>0$; and we do not require $f>0$. Notice also that we allow $f$ to depend on $(x, u)$; and that we do not require monotonicity, either on $f$, or on $s \rightarrow f(s) / s$. Our range of values of $\alpha$ is wider than the range allowed in [8]. On the other hand, we cannot guarantee that the solutions that we found are strictly positive in $\Omega$. Moreover, we obtain neither the uniqueness, nor the monotonicity obtained in [8]. Finally, our $\lambda^{*}$ does not have the optimality property of its counterpart in ([8], Theorem 2).

Our approach to study problem (1.1) is variational, and adapted from the one followed in [19]. Note that problem (1.1) has additional challenges with respect to the one considered in [19]: not only the nonlinearity is more general, but a further obstacle is posed by the fact that, 
when $\alpha \geq 1$, the domain of the corresponding energy functional $J$ is not an open subset of $H_{0}^{1}(\Omega)$. In order to circumvent this obstacle we will consider, for any $M>0$, the functional $J$ on the set $D_{M}^{\alpha}$ formed by the nonnegative functions $u \in H_{0}^{1}(\Omega)$ that are bounded by $M$, and such that $J(u)$ is well defined and finite. In Section 2 we prove that, on $D_{M}^{\alpha}, J$ has a nonnegative minimizer $u_{M} \not \equiv 0$; and that $\left\|u_{M}\right\|_{\infty} \leq \mathscr{M}$, with $\mathscr{M}$ constant and independent of $M$. From these facts, and some auxiliary lemmas, Theorem 1.2 is proved in Section 3 by showing that, for $M$ large enough, $u_{M}$ is a weak solution of (1.1) (in spite of the possible lack of differentiability of $J$ at $u_{M}$ ). Finally, at the end of Section 3, we use Theorem 1.2 to obtain Theorems 1.3 and 1.4.

\section{Preliminaries}

Let us recall that $\lambda \in \mathbb{R}$ is called a principal eigenvalue for $-\Delta$ in $\Omega$, with homogeneous Dirichlet boundary condition and weight function $b$, if the problem $-\Delta u=\lambda b u$ in $\Omega, u=0$ on $\partial \Omega$ has a solution $\phi$ such that $\phi>0$ in $\Omega$.

Remark 2.1. The following facts are well known (see e.g., [13]). If $\Omega$ is a $C^{1,1}$ domain in $\mathbb{R}^{n}$, $b \in L^{\infty}(\Omega)$ and $b^{+} \not \equiv 0$ then:

i) There exists a unique positive principal eigenvalue $\lambda_{1}(b)$, its eigenspace is one dimensional, and is included in $C^{1}(\bar{\Omega})$. Moreover, for each positive eigenfunction $\phi$, there are positive constants $c_{1}, c_{2}$ such that $c_{1} d_{\Omega} \leq \phi \leq c_{2} d_{\Omega}$ in $\Omega$. In particular, for $\gamma \in \mathbb{R}, \phi^{\gamma}$ is integrable if, and only if, $\gamma>-1$.

ii) If $0<\lambda<\lambda_{1}(b)$ and $h \in L^{\infty}(\Omega)$, the problem $-\Delta u=\lambda b u+h$ in $\Omega, u=0$ on $\partial \Omega$, has a unique solution $u \in \cap_{1 \leq p<\infty} W^{2, p}(\Omega)$, and the corresponding solution operator $(-\Delta-\lambda b)^{-1}: L^{\infty}(\Omega) \rightarrow C_{0}^{1}(\bar{\Omega})$ is bounded and strongly positive, i.e., if $h \in L^{\infty}(\Omega)$ and $0 \leq h \not \equiv 0$ then $u$ belongs to the interior of the positive cone of $C_{0}^{1}(\bar{\Omega})$ where $C_{0}^{1}(\bar{\Omega}):=\left\{v \in C^{1}(\bar{\Omega}): v=0\right.$ on $\left.\partial \Omega\right\}$.

iii) If $b^{*} \in L^{\infty}(\Omega)$ and $b \leq b^{*}$ then $\lambda_{1}\left(b^{*}\right) \leq \lambda_{1}(b)$.

For $M>0$ and $0<\alpha<3$, let $D_{M}^{\alpha} \subset H_{0}^{1}(\Omega)$ be defined by

$$
\begin{aligned}
& D_{M}^{\alpha}:=\left\{u \in H_{0}^{1}(\Omega): 0 \leq u \leq M\right\} \text { if } 0<\alpha<1, \\
& D_{M}^{\alpha}:=\left\{u \in H_{0}^{1}(\Omega): 0 \leq u \leq M \text { and } \int_{\{a>0\}} a|\ln u|<\infty\right\} \text { if } \alpha=1, \\
& D_{M}^{\alpha}:=\left\{u \in H_{0}^{1}(\Omega): 0 \leq u \leq M \text { and } \int_{\{a>0\}} a u^{1-\alpha}<\infty\right\} \text { if } 1<\alpha<3 .
\end{aligned}
$$

Lemma 2.2. Assume H1). Then $D_{M}^{\alpha} \neq \varnothing$ for any $M>0$ and $\alpha \in(0,3)$. 
Proof. The lemma is immediate when $0<\alpha<1$. For $1<\alpha<3$, we can proceed as follows: Let $\phi$ be a positive principal eigenfunction for $-\Delta$ in $\Omega$ with homogeneous Dirichlet boundary condition, with weight function 1 , and normalized such that $\|\phi\|_{\infty}=M^{\frac{1+\alpha}{2}}$. Note that $\left|\nabla\left(\phi^{\frac{2}{1+\alpha}}\right)\right|^{2}=\left(\frac{2}{1+\alpha}\right)^{2} \phi^{\frac{2(1-\alpha)}{1+\alpha}}|\nabla \phi|^{2}$ and that, since $\alpha<3$, we have $\frac{2(1-\alpha)}{1+\alpha}>-1$. Thus $\left|\nabla\left(\phi^{\frac{2}{1+\alpha}}\right)\right| \in L^{2}(\Omega)$. Clearly $\phi^{\frac{2}{1+\alpha}} \in L^{2}(\Omega)$ and $a \phi^{\frac{2(1-\alpha)}{1+\alpha}} \in L^{1}(\Omega)$, and then $\phi^{\frac{2}{1+\alpha}} \in D_{M}^{\alpha}$.

Consider now the case $\alpha=1$. Let $\delta \in(0,1)$ and let $\beta=1+\delta$. Thus $1<\beta<3$. Since $\lim _{s \rightarrow 0^{+}} \delta|\ln (s)|=0$, and $|\ln (s)|<s$ for $s>1$, there is a positive constant $c$ such that $|\ln (s)| \leq$ $c\left(s^{-\delta}+s\right)$ for any $s>0$. Let $\phi$ be a principal eigenfunction as above, but normalized now by $\|\phi\|_{\infty}=M^{\frac{1+\beta}{2}}$. As before, we have $\phi^{\frac{2}{1+\beta}} \in H_{0}^{1}(\Omega)$. Also, $\left|\ln \left(\phi^{\frac{2}{1+\beta}}\right)\right| \leq c\left(\phi^{-\frac{2 \delta}{1+\beta}}+\phi^{\frac{2}{1+\beta}}\right)=$ $c\left(\phi^{\frac{2(1-\beta)}{1+\beta}}+\phi^{\frac{2}{1+\beta}}\right)$. Since $\phi^{\frac{2(1-\beta)}{1+\beta}}$ and $\phi^{\frac{2}{1+\beta}}$ belong to $L^{1}(\Omega)$, it follows that $\int_{\Omega} a\left|\ln \left(\phi^{\frac{2}{1+\beta}}\right)\right|<$ $\infty$, and so $\phi^{\frac{2}{1+\beta}} \in D_{M}^{1}$.

For $0<\alpha<3$, let $J: \cup_{M>0} D_{M}^{\alpha} \rightarrow \mathbb{R}$ be defined by

$$
\begin{aligned}
& J(u):=\frac{1}{2} \int_{\Omega}|\nabla u|^{2}-\frac{1}{1-\alpha} \int_{\{a>0\}} a u^{1-\alpha}-\int_{\Omega} F(., u) \text { if } \alpha \neq 1, \\
& J(u):=\frac{1}{2} \int_{\Omega}|\nabla u|^{2}-\int_{\{a>0\}} a \ln (u)-\int_{\Omega} F(., u) \text { if } \alpha=1,
\end{aligned}
$$

where $F(x, s):=\int_{0}^{s} f(x, \sigma) d \sigma$.

Lemma 2.3. i) Assume H2) and H3). Let $M>0$, and let $\left\{u_{j}\right\}_{j \in \mathbf{N}}$ be a sequence of measurable functions on $\Omega$ such that $0 \leq u_{j} \leq M$ for all $j \in \mathbb{N}$, and $\lim _{j \rightarrow \infty} u_{j}=u$ a.e. in $\Omega$ for some $u: \Omega \rightarrow \mathbb{R}$. Then $\lim _{j \rightarrow \infty} \int_{\Omega} F\left(., u_{j}\right)=\int_{\Omega} F(., u)$.

ii) If H2) and H3) hold, and if $u, v$ are nonnegative functions in $L^{\infty}(\Omega)$, then

$$
\begin{aligned}
\lim _{t \rightarrow 0^{+}} \frac{1}{t} \int_{\Omega}(F(., u+t v)-F(., u)) & =\int_{\Omega} v f(., u) \text { and } \\
\lim _{t \rightarrow 0^{+}} \int_{\Omega}(F(., u+t v)-F(., u)) & =0 .
\end{aligned}
$$

If, in addition, $u-\varepsilon_{0} v \geq 0$ for some $\varepsilon_{0}>0$. then

$$
\begin{aligned}
\lim _{t \rightarrow 0^{-}} \frac{1}{t} \int_{\Omega}(F(., u+t v)-F(., u)) & =\int_{\Omega} v f(., u) \text { and } \\
\lim _{t \rightarrow 0^{-}} \int_{\Omega}(F(., u+t v)-F(., u)) & =0 .
\end{aligned}
$$

Proof. i) follows easily from $H 2$ ) and $H 3$ ) applying Lebesgue's dominated convergence theorem. 
To see ii) note that, for $0<t<1$, by the mean value theorem,

$$
F(., u+t v)-F(., u)=t v f\left(., u+\eta_{t}\right)
$$

in $\{v>0\}$; where $\eta_{t}:\{v>0\} \rightarrow \mathbb{R}$ depends on $u, v, t$, and satisfies $0 \leq \eta_{t} \leq t\|v\|_{\infty}$. We define $\eta_{t}=0$ in $\{v=0\}$, so that (2.6) holds in $\Omega$. Now,

$$
\begin{aligned}
& \left|\frac{1}{t} \int_{\Omega}(F(., u+t v)-F(., u))-\int_{\Omega} v f(., u)\right| \\
& =\left|\int_{\Omega} v\left(f\left(., u+\eta_{t}\right)-f(., u)\right)\right| \leq \int_{\Omega} v\left|f\left(., u+\eta_{t}\right)-f(., u)\right| .
\end{aligned}
$$

By $H 2), \lim _{t \rightarrow 0^{+}} v\left|f\left(., u+\eta_{t}\right)-f(., u)\right|=0$ a.e. in $\Omega$; and, by $\left.H 3\right)$,

$$
v\left|f\left(., u+\eta_{t}\right)-f(., u)\right| \leq 2 M \sup _{0 \leq s \leq 2 M}|f(., s)| \in L^{1}(\Omega),
$$

where $M:=\|u\|_{\infty}+\|v\|_{\infty}$. Then, by Lebesgue's dominated convergence theorem,

$$
\lim _{t \rightarrow 0^{+}} \int_{\Omega} v\left|f\left(., u+\eta_{t}\right)-f(., u)\right|=0 .
$$

Thus (2.2) (and so also (2.3)) holds. The proofs of (2.4) and (2.5) are similar.

Lemma 2.4. Assume H1)-H3), and let $M>0, \alpha \in(0,3)$. Then

i) $J$ is coercive on $D_{M}^{\alpha}$ with respect to the topology of $H_{0}^{1}(\Omega)$; i.e., $J(u) \rightarrow \infty$ when $u \in D_{M}^{\alpha}$, and $\|\nabla u\|_{2} \rightarrow \infty$.

ii) $\inf _{u \in D_{M}^{\alpha}} J(u)$ is achieved at some $u \in D_{M}^{\alpha}$.

Proof. For $u \in D_{M}^{\alpha}$ we have $\left|\int_{\Omega} F(., u)\right| \leq M B_{M}$, where $B_{M}:=\int_{\Omega} \sup _{0 \leq s \leq M}|f(., s)|$. Note that, by $H 3), B_{M}<\infty$.

If $1<\alpha<3$, we have $-\frac{1}{1-\alpha} \int_{\Omega} a u^{1-\alpha} \geq 0$, then $J(u) \geq \frac{1}{2} \int_{\Omega}|\nabla u|^{2}-M B_{M}$, which implies i).

If $0<\alpha<1$, from Hölder's and Poincaré's inequalities we get $\frac{1}{1-\alpha} \int_{\Omega} a u^{1-\alpha} \leq c\|\nabla u\|_{2}^{1-\alpha}$ for some positive constant $c$ independent of $u$. Thus $J(u) \geq \frac{1}{2}\|\nabla u\|_{2}^{2}-c\|\nabla u\|_{2}^{1-\alpha}-M B_{M}$; therefore i) holds also in this case.

If $\alpha=1$, using Poincaré's inequality, and that $\ln s \leq s$ for $s>0$, for some positive constant $c$ independent of $u$ we get

$$
-\int_{\{a>0\}} a \ln u \geq-\int_{\{a>0\} \cap\{u \geq 1\}} a \ln u \geq-\int_{\{a>0\} \cap\{u \geq 1\}} a u \geq-\int_{\Omega} a u \geq-c\|\nabla u\|_{2}
$$

and then $J(u) \geq \frac{1}{2}\|\nabla u\|_{2}^{2}-c\|\nabla u\|_{2}-B_{M}$; consequently i) holds when $\alpha=1$. 
To prove ii), let $\beta:=\inf _{u \in D_{M}^{\alpha}} J(u)$. Since $D_{M}^{\alpha} \neq \varnothing$, we have $\beta<\infty$. Consider a sequence $\left\{u_{j}\right\}_{j \in \mathbf{N}} \subset D_{M}^{\alpha}$ such that $\lim _{j \rightarrow \infty} J\left(u_{j}\right)=\beta$; it follows from i) that $\left\{u_{j}\right\}_{j \in \mathbf{N}}$ is bounded in $H_{0}^{1}(\Omega)$. Since the inclusion $H_{0}^{1}(\Omega) \hookrightarrow L^{2}(\Omega)$ is compact, there exist $u \in H_{0}^{1}(\Omega)$, and a subsequence $\left\{u_{j_{k}}\right\}_{k \in \mathbf{N}}$ such that $\left\{u_{j_{k}}\right\}_{k \in \mathbf{N}}$ converges strongly in $L^{2}(\Omega)$, and such that $\left\{\nabla u_{j_{k}}\right\}_{k \in \mathbb{N}}$ converges weakly to $\nabla u$ in $L^{2}\left(\Omega, \mathbf{R}^{n}\right)$. Taking a subsequence if necessary, we can assume that $\left\{u_{j_{k}}\right\}_{k \in \mathbf{N}}$ converges to $u$ a.e. in $\Omega$. Thus

$$
\|\nabla u\|_{2} \leq \underline{\lim }_{k \rightarrow \infty}\left\|\nabla u_{j_{k}}\right\|_{2} .
$$

Note that $u \in D_{M}^{\alpha}$. Indeed, since $0 \leq u_{j_{k}} \leq M$ for all $k$, we have $0 \leq u \leq M$, and so $u \in D_{M}^{\alpha}$ when $0<\alpha<1$. If $1<\alpha<3$, by Fatou's lemma,

$$
\begin{aligned}
-\frac{1}{1-\alpha} \int_{\Omega} a u^{1-\alpha} & \leq \varliminf_{k \rightarrow \infty} \int_{\Omega} \frac{-1}{1-\alpha} a u_{j_{k}}^{1-\alpha} \\
& =\underline{\lim }_{k \rightarrow \infty}\left(J\left(u_{j_{k}}\right)-\frac{1}{2} \int_{\Omega}\left|\nabla u_{j_{k}}\right|^{2}+\int_{\Omega} F\left(., u_{j_{k}}\right)\right) \\
& \leq \varliminf_{k \rightarrow \infty} J\left(u_{j_{k}}\right)+M B_{M}<\infty
\end{aligned}
$$

and then $u \in D_{M}^{\alpha}$ when $1<\alpha<3$. If $\alpha=1$, again by Fatou's Lemma,

$$
\begin{aligned}
\int_{\{a>0\}} a|\ln u| & =\int_{\{a>0\}} \underline{\lim _{k \rightarrow \infty} a\left|\ln u_{j_{k}}\right|} \\
& \leq \underline{\lim }_{k \rightarrow \infty}\left(-\int_{\{a>0\} \cap\left\{u_{j_{k}} \leq 1\right\}} a \ln u_{j_{k}}+\int_{\{a>0\} \cap\left\{u_{j_{k}}>1\right\}} a \ln u_{j_{k}}\right) \\
& =\underline{\lim }_{k \rightarrow \infty}\left(-\int_{\{a>0\}} a \ln u_{j_{k}}+2 \int_{\{a>0\} \cap\left\{u_{j_{k}}>1\right\}} a \ln u_{j_{k}}\right) \\
& \leq \underline{\lim }_{k \rightarrow \infty}\left(-\int_{\{a>0\}} a \ln u_{j_{k}}+2 \int_{\{a>0\}} a u_{j_{k}}\right)
\end{aligned}
$$

and, since $\left\{a u_{j_{k}}\right\}_{k \in \mathbb{N}}$ converges to $a u$ in the $L^{1}(\Omega)$ norm,

$$
\begin{aligned}
& \underline{\lim }_{k \rightarrow \infty}\left(-\int_{\{a>0\}} a \ln u_{j_{k}}+2 \int_{\{a>0\}} a u_{j_{k}}\right) \\
& =\underline{\lim }_{k \rightarrow \infty}\left(-\int_{\{a>0\}} a \ln u_{j_{k}}\right)+2 \int_{\{a>0\}} a u \\
& =\underline{\lim }_{k \rightarrow \infty}\left(J\left(u_{j_{k}}\right)-\frac{1}{2} \int_{\Omega}\left|\nabla u_{j_{k}}\right|^{2}+\int_{\Omega} F\left(., u_{j_{k}}\right)\right)+2 \int_{\{a>0\}} a u \\
& \leq \varliminf_{k \rightarrow \infty} J\left(u_{j_{k}}\right)+M B_{M}+2 \int_{\{a>0\}} a u<\infty .
\end{aligned}
$$


Then $u \in D_{M}^{\alpha}$ also when $\alpha=1$. Since $u \in D_{M}^{\alpha}$, we have $J(u) \geq \beta$; therefore, to prove ii), it remains to show that $J(u) \leq \beta$. To do this observe that, by Lemma $2.3 \mathrm{i}$ ),

$$
\lim _{k \rightarrow \infty} \int_{\Omega} F\left(., u_{j_{k}}\right)=\int_{\Omega} F(., u) .
$$

If $1<\alpha<3$, from (2.7), (2.8) and Fatou's lemma, we get

$$
\begin{aligned}
J(u) & =\frac{1}{2} \int_{\Omega}|\nabla u|^{2}-\frac{1}{1-\alpha} \int_{\{a>0\}} a u^{1-\alpha}-\int_{\Omega} F(., u) \\
& \leq \underline{\lim }_{k \rightarrow \infty}\left(\frac{1}{2} \int_{\Omega}\left|\nabla u_{j_{k}}\right|^{2}\right)+\underline{\lim }_{k \rightarrow \infty}\left(-\frac{1}{1-\alpha} \int_{\{a>0\}} a u_{j_{k}}^{1-\alpha}\right) \\
& +\lim _{k \rightarrow \infty} \int_{\Omega} F\left(., u_{j_{k}}\right) \\
& \leq \underline{\lim }_{k \rightarrow \infty}\left(\frac{1}{2} \int_{\Omega}\left|\nabla u_{j_{k}}\right|^{2}-\frac{1}{1-\alpha} \int_{\{a>0\}} a u^{1-\alpha}-\int_{\Omega} F\left(., u_{j_{k}}\right)\right) \\
& =\underline{\lim }_{k \rightarrow \infty} J\left(u_{j_{k}}\right)=\beta .
\end{aligned}
$$

Then $J(u) \leq \beta$ when $1<\alpha<3$. Consider now the case $0<\alpha<1$ : since $0 \leq u_{j_{k}} \leq M$ for all $k$, Lebesgue's dominated convergence gives $\lim _{k \rightarrow \infty} \frac{1}{1-\alpha} \int_{\{a>0\}} a u_{j_{k}}^{1-\alpha}=\frac{1}{1-\alpha} \int_{\{a>0\}} a u^{1-\alpha}$, and then, as in (2.9), we get $J(u) \leq \beta$.

Finally suppose $\alpha=1$ : Since $u_{j_{k}} \in D_{M}^{\alpha}$ we have $a \ln \left(M / u_{j_{k}}\right) \geq 0$, and then Fatou's lemma gives

$$
\begin{aligned}
-\int_{\{a>0\}} a \ln u & =\int_{\{a>0\}} a \ln \left(\frac{M}{u}\right)-\int_{\{a>0\}} a \ln M \\
& =\int_{\{a>0\}} \underline{\lim } k \rightarrow \infty \\
& \leq \underline{\lim }_{k \rightarrow \infty} \int_{\{a>0\}} a \ln \left(\frac{M}{u_{j_{k}}}\right)-\int_{\{a>0\}} a \ln M \\
& =\underline{\lim }_{k \rightarrow \infty}\left(\int_{\{a>0\}} a \ln M-\int_{\{a>0\}} a \ln M\right. \\
& =\underline{\lim }_{k \rightarrow \infty}\left(-\int_{\{a>0\}} a \ln u_{j_{k}}\right)-\int_{\{a>0\}} a \ln M
\end{aligned}
$$

Now, we proceed as in (2.9), replacing there $-\frac{1}{1-\alpha} \int_{\{a>0\}} a u_{j_{k}}^{1-\alpha}$ by $-\int_{\{a>0\}} a \ln u_{j_{k}}$, and $-\frac{1}{1-\alpha} \int_{\{a>0\}} a u^{1-\alpha}$ by $-\int_{\{a>0\}} a \ln u$, to conclude that $J(u) \leq \beta$ also for $\alpha=1$.

Lemma 2.5. Assume H1)-H3), and let $M>0, \alpha \in(0,3)$. Then

$$
\int_{\Omega}\langle\nabla u, \nabla(u \varphi)\rangle \leq \int_{\Omega} \chi_{\{u>0\}} a u^{1-\alpha} \varphi+\int_{\Omega} f(., u) u \varphi
$$


for any minimizer $u$ for $J$ on $D_{M}^{\alpha}$, and for any nonnegative $\varphi \in H^{1}(\Omega) \cap L^{\infty}(\Omega)$.

Proof. Let $u$ be a minimizer for $J$ on $D_{M}^{\alpha}, \tau \in(-1,0)$; and let $\varphi$ be a nonnegative function in $H^{1}(\Omega) \cap L^{\infty}(\Omega)$ that, in addition, satisfies $\|\varphi\|_{\infty} \leq \frac{1}{2}$.

Note that $u+\tau u \varphi \in D_{M}^{\alpha}$. Indeed, $0 \leq u+\tau u \varphi \leq M$ and (since $\left.u \in L^{\infty}(\Omega)\right) u+\tau u \varphi \in H_{0}^{1}(\Omega)$. In particular, this gives $u+\tau u \varphi \in D_{M}^{\alpha}$ when $0<\alpha<1$.

If $1<\alpha<3$ we have also $\left|a(u+\tau u \varphi)^{1-\alpha}\right| \leq \frac{1}{2^{1-\alpha}} a u^{1-\alpha} \in L^{1}(\{a>0\})$, and so $u+\tau u \varphi \in$ $D_{M}^{\alpha}$.

If $\alpha=1$ then $|a \ln (u+\tau u \varphi)|=a|\ln u+\ln (1+\tau \varphi)| \leq a|\ln u|+a|\ln (1+\tau \varphi)| \in L^{1}(\{a>0\})$ and so, again in this case, $u+\tau u \varphi \in D_{M}^{\alpha}$.

To prove (2.10) we consider first the case where $\alpha \neq 1$. Since $J(u) \leq J(u+\tau u \varphi)$, a computation gives

$$
\begin{aligned}
& \tau \int_{\Omega}\langle\nabla u, \nabla(u \varphi)\rangle \\
& \geq \frac{1}{1-\alpha} \int_{\{a>0\}} a u^{1-\alpha}\left((1+\tau \varphi)^{1-\alpha}-1\right)+\int_{\Omega}(F(., u+\tau \varphi u)-F(., u)) \\
& -\frac{\tau^{2}}{2} \int_{\Omega} u^{2}|\nabla \varphi|^{2}-\frac{\tau^{2}}{2} \int_{\Omega} \varphi^{2}|\nabla u|^{2}-\tau^{2} \int_{\Omega} u \varphi\langle\nabla u, \nabla \varphi\rangle,
\end{aligned}
$$

and a Taylor expansion gives

$$
(1+\tau \varphi)^{1-\alpha}-1=(1-\alpha) \tau \varphi+\frac{\tau^{2}}{2}(1-\alpha) \alpha(1+\zeta)^{-\alpha-1} \varphi^{2}
$$

for some measurable function $\zeta$ such that $-\frac{1}{2} \leq \tau \varphi \leq \zeta \leq 0$. Since $a u^{1-\alpha} \in L^{1}(\{a>0\})$, and $1+\zeta \geq \frac{1}{2}$, we have $\left|\int_{\{a>0\}} a u^{1-\alpha}(1+\zeta)^{-\alpha-1} \varphi^{2}\right| \leq c$ where $c$ is a positive constant independent of $\tau$; and so,

$$
\lim _{\tau \rightarrow 0^{-}} \frac{1}{(1-\alpha) \tau} \int_{\{a>0\}} a u^{1-\alpha}\left((1+\tau \varphi)^{1-\alpha}-1\right)=\int_{\{a>0\}} a u^{1-\alpha} \varphi .
$$

Also, by Lemma 2.3 ii) we have

$$
\lim _{\tau \rightarrow 0^{-}} \frac{1}{\tau} \int_{\Omega}(F(., u+\tau \varphi u)-F(., u))=\int_{\Omega} \varphi u f(., u) .
$$

Dividing by $\tau$ the inequality (2.11), letting $\tau \rightarrow 0^{-}$, and using (2.12) and (2.13), we get

$$
\int_{\Omega}\langle\nabla u, \nabla(u \varphi)\rangle \leq \int_{\{a>0\}} a u^{1-\alpha} \varphi+\int_{\Omega} f(., u) u \varphi .
$$

Note that $a u^{1-\alpha} \varphi=\chi_{\{u>0\}} a u^{1-\alpha} \varphi$ (this clearly holds when $0<\alpha<1$; and when $1 \leq \alpha<3$ the equality follows from the fact that $u>0$ a.e. in $\{a>0\}$ ). Thus (2.14) gives (2.10) and, 
since both sides in (2.10) are linear on $\varphi$, our additional assumption $\|\varphi\|_{\infty} \leq \frac{1}{2}$ can be removed. Thus the lemma holds when $\alpha \neq 1$.

If $\alpha=1$ we have, as before, (2.11), with the term $\frac{1}{1-\alpha} \int_{\{a>0\}} a u^{1-\alpha}\left((1+\tau \varphi)^{1-\alpha}-1\right)$ replaced by $\int_{\{a>0\}} a(\ln (u(1+\tau \varphi))-\ln u)=\int_{\{a>0\}} a \ln (1+\tau \varphi)$; and a Taylor expansion gives $\ln (1+\tau \varphi)=\tau \varphi-\left(1+\zeta_{\tau}\right)^{-2} \tau^{2} \varphi^{2}$ for some measurable function $\zeta_{\tau}: \Omega \rightarrow \mathbb{R}$ satisfying $-\frac{1}{2} \leq \tau \varphi \leq \zeta_{\tau} \leq 0$. Then

$$
\lim _{\tau \rightarrow 0^{-}} \frac{1}{\tau} \int_{\{a>0\}} a(\ln (u(1+\tau \varphi))-\ln u)=\int_{\{a>0\}} a u \varphi
$$

and so, proceeding as in the previous case, we conclude that (2.10) holds when $\|\varphi\|_{\infty} \leq \frac{1}{2}$; and, as before, this additional assumption on $\varphi$ can be removed.

Lemma 2.6. Assume H1)-H3). Let $M>0, \alpha \in(0,3)$. Let $m$ be an integer such that $m \geq$ $\max \{2,1+\alpha\}$, and let $u$ be a minimizer for $J$ on $D_{M}^{\alpha}$. Then, for any nonnegative $\varphi \in H_{0}^{1}(\Omega) \cap$ $L^{\infty}(\Omega)$,

$$
\int_{\Omega}\left\langle\nabla\left(u^{m}\right), \nabla(\varphi)\right\rangle \leq m \int_{\Omega}\left(a u^{m-1-\alpha}+u^{m-1} f(., u)\right) \varphi
$$

Proof. $u$ is bounded, therefore $u^{m} \in H_{0}^{1}(\Omega)$ and $\nabla\left(u^{m}\right)=m u^{m-1} \nabla u$. Also $u^{m-2} \varphi \in H_{0}^{1}(\Omega) \cap$ $L^{\infty}(\Omega)$ for any nonnegative $\varphi \in H_{0}^{1}(\Omega) \cap L^{\infty}(\Omega)$. Then

$$
\begin{aligned}
& \int_{\Omega}\left\langle\nabla\left(u^{m}\right), \nabla \varphi\right\rangle \\
& =m \int_{\Omega} u^{m-1}\langle\nabla u, \nabla \varphi\rangle \\
& =m \int_{\Omega}\left\langle\nabla u, \nabla\left(u^{m-1} \varphi\right)\right\rangle-m(m-1) \int_{\Omega} u^{m-2} \varphi|\nabla u|^{2} \\
& \leq m \int_{\Omega}\left(\chi_{\{u>0\}} a u^{m-1-\alpha}+u^{m-1} f(., u)\right) \varphi
\end{aligned}
$$

the last inequality by Lemma 2.5. Since $\chi_{\{u>0\}} a u^{m-1-\alpha}=a u^{m-1-\alpha}$, the lemma follows.

Remark 2.7. Let $u \in L_{l o c}^{1}(\Omega)$ such that $\nabla u \in L^{2}(\Omega)$, and let $w \in L^{\infty}(\Omega)$. If $\int_{\Omega}\langle\nabla u, \nabla \varphi\rangle \leq$ $\int_{\Omega} w \varphi$ (respectively $\int_{\Omega}\langle\nabla u, \nabla \varphi\rangle \geq \int_{\Omega} w \varphi$ ) for every nonnegative $\varphi \in H_{0}^{1}(\Omega) \cap L^{\infty}(\Omega)$ then the corresponding inequality holds for all nonnegative $\varphi \in H_{0}^{1}(\Omega)$ (by using a density argument with the truncations $\left.\varphi_{j}(x):=\min \{\varphi(x), j\}, j \in \mathbb{N}\right)$.

Lemma 2.8. Assume H1)-H4), and $\alpha \in(0,3)$. Then there exists a positive number $\mathscr{M}$ such that, for any $M>0$, and any minimizer $u$ for $J$ on $D_{M}^{\alpha},\|u\|_{\infty} \leq \mathscr{M}$. 
Proof. Let $M>0$, and let $u$ be a minimizer for $J$ on $D_{M}^{\alpha}$. Assume first that $H 4^{\prime}$ ) holds, and let $m$ and $b$ be as there. By Lemma 2.6, for $0 \leq \varphi \in H_{0}^{1}(\Omega) \cap L^{\infty}(\Omega)$,

$$
\begin{aligned}
\int_{\Omega}\left\langle\nabla\left(u^{m}\right), \nabla(\varphi)\right\rangle & \leq m \int_{\Omega}\left(\chi_{\{u>0\}} a u^{m-1-\alpha}+u^{m-1} f(., u)\right) \varphi \\
& \leq m \int_{\Omega}\left(\chi_{\{u>0\}} a u^{m-1-\alpha}+b u^{m}\right) \varphi
\end{aligned}
$$

and so, by Remark 2.7, the same inequalities hold for all nonnegative $\varphi \in H_{0}^{1}(\Omega)$, i.e.,

$$
(-\Delta-m b)\left(u^{m}\right) \leq m \chi_{\{u>0\}} a u^{m-1-\alpha}=m a u^{m-1-\alpha} \text { in }\left(H_{0}^{1}(\Omega)\right)^{\prime} .
$$

Since $0<m<\lambda_{1}(b)$, the operator $(-\Delta-m b)^{-1}: L^{\infty}(\Omega) \rightarrow H_{0}^{1}(\Omega) \subset L^{\infty}(\Omega)$ is well defined, bounded and positive. Let $v:=(-\Delta-m b)^{-1}\left(m^{m-1-\alpha}\right)$. Then

$$
\|u\|_{\infty}^{m} \leq\|v\|_{\infty} \leq\left\|(-\Delta-m b)^{-1}\right\|_{\infty, \infty}\left\|m a u^{m-1-\alpha}\right\|_{\infty}=c\|u\|_{\infty}^{m-1-\alpha}
$$

for some positive constant $c$ independent of $M$ and $u$; therefore the lemma holds with $\mathscr{M}=c^{\frac{1}{1+\alpha}}$.

Assume now that $\left.H 4^{\prime \prime}\right)$ holds. Let $m$ be an integer such that $m \geq \max \{2,1+\alpha\}$, let $\lambda_{1}:=$ $\lambda_{1}(\mathbf{1})$, let $\varepsilon \in\left(0, \frac{\lambda_{1}}{m}\right)$, and let $s_{0}>0$ be such that $\sup _{s \geq s_{0}} \frac{f(., s)}{s} \leq \varepsilon$. From Lemma 2.6 we have, for $0 \leq \varphi \in H_{0}^{1}(\Omega) \cap L^{\infty}(\Omega)$, that

$$
\begin{aligned}
& \int_{\Omega}\left\langle\nabla\left(u^{m}\right), \nabla(\varphi)\right\rangle \\
& \leq m \int_{\Omega} \chi_{\{u>0\}} a u^{m-1-\alpha} \varphi+m \int_{\left\{u<s_{0}\right\}} u^{m-1} f(., u) \varphi+m \int_{\left\{u \geq s_{0}\right\}} u^{m-1} f(., u) \varphi \\
& \leq m \int_{\Omega} \chi_{\{u>0\}} a u^{m-1-\alpha} \varphi+m s_{0}^{m-1} \int_{\left\{u<s_{0}\right\}} \sup _{s<s_{0}}|f(., s)| \varphi+m \int_{\left\{u \geq s_{0}\right\}} u^{m} \frac{f(., u)}{u} \varphi \\
& \leq m \int_{\Omega}\left(a u^{m-1-\alpha}+A+m \varepsilon u^{m}\right) \varphi
\end{aligned}
$$

where

$$
A:=m s_{0}^{m-1} \sup _{(x, s) \in \bar{\Omega} \times\left[0, s_{0}\right]}|f(x, s)|
$$

is a constant independent of $M$ and $u$. Thus, by Remark 2.7,

$$
-\Delta\left(u^{m}\right) \leq m a\left(u^{m}\right)^{\frac{m-1-\alpha}{m}}+m \varepsilon u^{m}+A \text { in }\left(H_{0}^{1}(\Omega)\right)^{\prime} .
$$

Since $m \varepsilon<\lambda_{1}(\mathbf{1})$ we have that $(-\Delta-m \varepsilon \mathbf{1})^{-1}$ is a bounded and positive operator on $L^{\infty}(\Omega)$; and so, from (2.17), $u^{m} \leq(-\Delta-m \varepsilon \mathbf{1})^{-1}\left(m a\left(u^{m}\right)^{\frac{m-1-\alpha}{m}}+A\right)$; which gives

$$
\left\|u^{m}\right\|_{\infty} \leq c^{\prime}\left\|u^{m}\right\|_{\infty}^{\frac{m-1-\alpha}{m}}+c^{\prime}
$$


for some $c^{\prime}$ independent of $M$ and $u$. Since $0<\frac{m-1-\alpha}{m}<1$, the lemma follows.

Remark 2.9. Let $w \in L^{1}(\Omega)$ such that $|\{w>0\}|>0$, and let $\beta \in[0,1)$. Then there exists a nonnegative $\Phi \in C_{c}^{\infty}(\Omega)$ such that $\int w \Phi^{1-\beta}>0$. Indeed, consider a nonnegative radial function $h \in C_{c}^{\infty}\left(\mathbb{R}^{n}\right)$ with support in the unit ball $B=\left\{x \in \mathbb{R}^{n}:|x|<1\right\}$ and such that $\int_{B} h=1$. For $\varepsilon>0$ let $h_{\varepsilon}(x):=\frac{1}{\varepsilon^{n}} h\left(\frac{x}{\varepsilon}\right)$ and for $\delta>0$ let $\Omega_{\delta}:=\{x \in \Omega: \operatorname{dist}(x, \partial \Omega)>\delta\}$. Then $\left|\{w>0\} \cap \Omega_{\delta}\right|>0$ for $\delta$ positive and small enough. Fix such a $\delta$ and define $E=\{x \in \Omega: w(x)>0\} \cap \Omega_{\delta}$. For $\varepsilon>0$ define $\Phi_{\varepsilon}:=h_{\varepsilon} * \chi_{E}$. Then $\Phi_{\varepsilon} \in C_{c}^{\infty}\left(\mathbb{R}^{n}\right)$ and $\operatorname{supp}\left(\Phi_{\varepsilon}\right) \subset \Omega$ for $\varepsilon<\delta$. Also, $\lim _{\varepsilon \rightarrow 0^{+}} \Phi_{\varepsilon}=\chi_{E}$ with convergence in $L^{1}(\Omega)$ (see e.g., [2], Theorem 4.22), and so $\lim _{j \rightarrow \infty} \Phi_{\varepsilon_{j}}=\chi_{E}$ a.e. in $\Omega$ for some sequence $\left\{\varepsilon_{j}\right\}_{j \in \mathbb{N}}$ such that $\lim _{j \rightarrow \infty} \varepsilon_{j}=0$. Then, by Fatou's Lemma, $0<\int_{\Omega} w \chi_{E} \leq \underline{\lim }_{j \rightarrow \infty} \int_{\Omega} w \Phi_{\varepsilon_{j}}^{1-\beta}$. Thus $\int_{\Omega} w \Phi_{\varepsilon_{j}}^{1-\beta}>0$ for $j$ large enough.

Lemma 2.10. Let $\alpha \in(0,3)$. Assume H1)-H4). Let $\mathscr{M}$ be as in Lemma 2.8, and let $M>\mathscr{M}$. If $u$ is a minimizer for $J$ on $D_{M}^{\alpha}$, then $\chi_{\{u>0\}} a u^{-\alpha} \not \equiv 0$. In particular, $u \neq \equiv 0$.

Proof. If $1 \leq \alpha<3, u \in D_{M}^{\alpha}$ implies $u>0$ a.e. in $\{a>0\}$, and so $\chi_{\{u>0\}} a u^{-\alpha} \not \equiv 0$. To prove the lemma when $0<\alpha<1$ we proceed by contradiction. Suppose that $u$ is a minimizer for $J$ on $D_{M}^{\alpha}$ and that $\chi_{\{u>0\}} a u^{-\alpha}=0$. Let $\Phi \in C_{c}^{\infty}(\Omega)$ such that $\Phi \geq 0$ and $\int a \Phi^{1-\alpha}>0$. By Lemma 2.8, $u \leq \mathscr{M}<M$; thus $u+t \Phi \in D_{M}^{\alpha}$ for $t$ positive and small enough, and so $J(u) \leq$ $J(u+t \Phi)$. Also, $\chi_{\{u>0\}} a u^{-\alpha}=0$ implies that $u=0$ a.e. in $\{a>0\}$. Then $\int_{\Omega} a u^{1-\alpha}=0$, and $\int_{\Omega} a(u+t \Phi)^{1-\alpha}=\int_{\Omega} a(t \Phi)^{1-\alpha}$. Thus the inequality $J(u) \leq J(u+t \Phi)$ can be written as

$$
0 \leq t \int_{\Omega}\langle\nabla u, \nabla \Phi\rangle+\frac{t^{2}}{2} \int_{\Omega}|\nabla \Phi|^{2}-\frac{t^{1-\alpha}}{1-\alpha} \int_{\{a>0\}} a \Phi^{1-\alpha}-\int_{\Omega}(F(., u+t \Phi)-F(., u)) .
$$

From this inequality, dividing by $t^{1-\alpha}$, taking the limit as $t \rightarrow 0^{+}$, using that, by Lemma 2.3 ii), $\lim _{t \rightarrow 0^{+}} \frac{1}{t} \int_{\Omega}(F(., u+t \Phi)-F(., u))=\int_{\Omega} \Phi f(., u)$, and recalling that $\int_{\{a>0\}} a \Phi^{1-\alpha}>0$, we obtain a contradiction.

In order to emphasize the dependence on $f$, we will sometimes write $J_{f}$ for the functional $J$.

Lemma 2.11. Let $\alpha \in(0,3)$. Assume H1)-H3), H5), and that either H6) or H7) holds. When

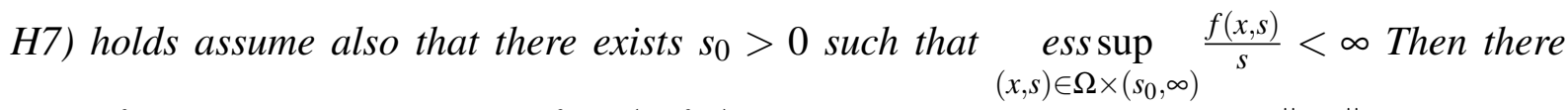
exists $\lambda^{*}>0$ such that, for any $\lambda \in\left(0, \lambda^{*}\right)$, there exists $\mathscr{M}_{\lambda}>0$ such that $\left\|u_{\lambda}\right\|_{\infty} \leq \mathscr{M}_{\lambda}$ for any $M>0$, and any minimizer $u_{\lambda}$ for $J_{\lambda f}$ on $D_{M}^{\alpha}$. If, in addition, $f \leq 0$ in $\Omega \times(0, \infty)$, then $\lambda^{*}=\infty$. 
Proof. Consider the case when $H 7$ ) holds. Let $M>0$ and let $u$ be a minimizer for $J_{\lambda f}$ on $D_{M}^{\alpha}$. Let $m$ be an integer such that $m \geq \max \{2,1+\alpha\}$ and let $k>\max \left\{0, \underset{x \in \Omega \times\left(s_{0}, \infty\right)}{\operatorname{ess} \sup } \frac{f(x, s)}{s}\right\}$. For $\lambda>0$ we can repeat the computations performed in (2.16), with $\lambda f$ and $\lambda m k$ in place of $f$ and $m \varepsilon$ respectively, to obtain, for $0 \leq \varphi \in H_{0}^{1}(\Omega) \cap L^{\infty}(\Omega)$, that

$$
\begin{aligned}
\int_{\Omega}\left\langle\nabla\left(u^{m}\right), \nabla(\varphi)\right\rangle & \leq m \int_{\Omega} a u^{m-1-\alpha} \varphi+A \int_{\Omega} \varphi+\int_{\Omega \cap\left\{u>s_{0}\right\}} m \lambda u^{m} \frac{f(x, u)}{u} \varphi \\
& \leq m \int_{\Omega} a u^{m-1-\alpha} \varphi+A \int_{\Omega} \varphi+\delta \int_{\Omega} m \lambda k u^{m} \varphi,
\end{aligned}
$$

where $\delta:=0$ if $f \leq 0$ in $\Omega \times[0, \infty)$, and $\delta:=1$ otherwise; and where

$$
A:=m \lambda s_{0}^{m-1}\left\|f_{\mid \Omega \times\left(0, s_{0}\right)}\right\|_{L^{\infty}\left(\Omega \times\left(0, s_{0}\right)\right)}
$$

is a constant independent of $M$ and $u$. Then, as in Lemma 2.8, we arrive to

$$
-\Delta\left(u^{m}\right) \leq m a\left(u^{m}\right)^{\frac{m-1-\alpha}{m}}+\delta m \lambda k u^{m}+A \text { in }\left(H_{0}^{1}(\Omega)\right)^{\prime} .
$$

If $\delta=1$ and $0<\lambda<\frac{\lambda_{1}(\mathbf{1})}{m k}$, then $\lambda_{1}(\lambda \delta m k \mathbf{1})=\frac{\lambda_{1}(\mathbf{1})}{\lambda m k}>1$; and so, from (2.21),

$$
u^{m} \leq(-\Delta-\lambda m k)^{-1}\left(m a\left(u^{m}\right)^{\frac{m-1-\alpha}{m}}+A\right),
$$

which implies (2.18) for some positive constant $c^{\prime}$ independent of $M$ and $u$; therefore the lemma holds with $\lambda^{*}=\frac{\lambda_{1}(\mathbf{1})}{m}$. If $\delta=0$ (i.e., if $\left.f \leq 0\right)$, (2.21) gives $u^{m} \leq(-\Delta)^{-1}\left(m a\left(u^{m}\right)^{\frac{m-1-\alpha}{m}}+A\right)$, which implies that (2.18) holds for all $\lambda \geq 0$; therefore, in this case, the lemma holds with $\lambda^{*}=\infty$.

When H6) holds the proof is similar: let $k>\max \left\{0, \underset{x \in \Omega \times(0, \infty)}{\operatorname{eesssup}} \frac{f(x, s)}{s}\right\}$. Instead of (2.20) we now have

$$
\begin{aligned}
\int_{\Omega}\left\langle\nabla\left(u^{m}\right), \nabla(\varphi)\right\rangle & \leq m \int_{\Omega} a u^{m-1-\alpha} \varphi+\delta \int_{\Omega \cap\{u>0\}} m \lambda u^{m} \frac{f(x, u)}{u} \varphi \\
& \leq m \int_{\Omega} a u^{m-1-\alpha} \varphi+\delta \int_{\Omega} m \lambda k u^{m} \varphi
\end{aligned}
$$

with $\delta$ as before. Thus (2.21) holds with $A=0$, and the proof ends as in the previous case.

\section{Proofs of the main results}

Proof of Theorem 1.2. Let $\mathscr{M}$ be as given in Lemma 2.8. Let $M=\mathscr{M}+1$, and let $u$ be a minimizer for $J$ on $D_{M}^{\alpha}$. Thus, by Lemma $2.10, \chi_{\{u>0\}} a u^{-\alpha} \not \equiv 0$ (and so $u \not \equiv 0$ ). Let $\psi$ be 
a nonnegative function in $H_{0}^{1}(\Omega) \cap L^{\infty}(\Omega)$, and let $\varepsilon>0$. Thus $\frac{\psi}{u+\varepsilon} \in H^{1}(\Omega) \cap L^{\infty}(\Omega)$, and $\nabla\left(u \frac{\psi}{u+\varepsilon}\right)=\varepsilon \frac{\nabla u}{(u+\varepsilon)^{2}} \psi+\frac{u}{u+\varepsilon} \nabla \psi$. Then Lemma 2.5 gives

$$
\begin{aligned}
& \varepsilon \int_{\Omega} \psi \frac{|\nabla u|^{2}}{(u+\varepsilon)^{2}}+\int_{\Omega} \frac{u}{u+\varepsilon}\langle\nabla u, \nabla \psi\rangle \\
& \leq \int_{\Omega} \chi_{\{a>0\}} a u^{1-\alpha} \frac{\psi}{u+\varepsilon}+\int_{\Omega} f(., u) u \frac{\psi}{u+\varepsilon} .
\end{aligned}
$$

Since $\nabla u=0$ a.e. in $\{u=0\},(3.1)$ can be written as

$$
\begin{aligned}
& \varepsilon \int_{\{u>0\}} \psi \frac{|\nabla u|^{2}}{(u+\varepsilon)^{2}}+\int_{\{u>0\}} \frac{u}{u+\varepsilon}\langle\nabla u, \nabla \psi\rangle \\
& -\int_{\{u>0\}} f(., u) \frac{u}{u+\varepsilon} \psi \leq \int_{\{u>0\}} a u^{-\alpha} \frac{u}{u+\varepsilon} \psi .
\end{aligned}
$$

Also $\lim _{\varepsilon \rightarrow 0^{+}} \frac{u}{u+\varepsilon}\langle\nabla u, \nabla \psi\rangle=\chi_{\{u>0\}}\langle\nabla u, \nabla \psi\rangle=\langle\nabla u, \nabla \psi\rangle$ a.e. in $\Omega$, and $\left|\frac{u}{u+\varepsilon}\langle\nabla u, \nabla \psi\rangle\right| \leq$ $|\langle\nabla u, \nabla \psi\rangle| \in L^{1}(\Omega)$, and so Lebesgue's dominated convergence theorem gives

$$
\lim _{\varepsilon \rightarrow 0^{+}} \int_{\{u>0\}} \frac{u}{u+\varepsilon}\langle\nabla u, \nabla \psi\rangle=\int_{\Omega}\langle\nabla u, \nabla \psi\rangle .
$$

Since $\lim _{\varepsilon \rightarrow 0^{+}} a u^{-\alpha} \frac{u}{u+\varepsilon} \psi=a u^{-\alpha} \psi$ a.e. in $\{u>0\}$, and $a u^{-\alpha} \frac{u}{u+\varepsilon} \psi$ is nonincreasing in $\varepsilon$, the monotone convergence theorem gives

$$
\lim _{\varepsilon \rightarrow 0^{+}} \int_{\{u>0\}} a u^{-\alpha} \frac{u}{u+\varepsilon} \psi=\int_{\{u>0\}} a u^{-\alpha} \psi=\int_{\Omega} \chi_{\{u>0\}} a u^{-\alpha} \psi
$$

Also, $\left|\frac{u}{u+\varepsilon} f(., u) \psi\right| \leq \sup _{0 \leq s \leq M}|f(., s)| \psi \in L^{1}(\Omega)$ and then, by Lebesgue's dominated convergence theorem,

$$
\lim _{\varepsilon \rightarrow 0^{+}} \int_{\{u>0\}} f(., u) \frac{u}{u+\varepsilon} \psi=\int_{\Omega} \chi_{\{u>0\}} f(., u) \psi \leq \int_{\Omega} f(., u) \psi,
$$

the last equality because, by $H 5), f(., 0) \geq 0$. Then, from (3.2), (3.3), (3.4) and (3.5), we have

$$
\begin{aligned}
& \int_{\Omega}\langle\nabla u, \nabla \psi\rangle-\int_{\Omega} f(., u) \psi \\
& \leq \int_{\Omega}\langle\nabla u, \nabla \psi\rangle-\int_{\Omega} \chi_{\{u>0\}} f(., u) \psi \\
& \leq \lim _{\varepsilon \rightarrow 0^{+}}\left(\int_{\{u>0\}} \frac{u}{u+\varepsilon}\langle\nabla u, \nabla \psi\rangle-\int_{\{u>0\}} f(., u) \frac{u}{u+\varepsilon} \psi\right) \\
& \leq \varlimsup_{\mathcal{E} \rightarrow 0^{+}}\left(\int_{\{u>0\}} \frac{\varepsilon \psi|\nabla u|^{2}}{(u+\varepsilon)^{2}}+\int_{\{u>0\}} \frac{u}{u+\varepsilon}\langle\nabla u, \nabla \psi\rangle-\int_{\{u>0\}} f(., u) \frac{u}{u+\varepsilon} \psi\right) \\
& \leq \varlimsup_{\mathcal{L} \rightarrow 0^{+}} \int_{\{u>0\}} a u^{-\alpha} \frac{u}{u+\varepsilon} \psi=\int_{\Omega} \chi_{\{u>0\}} a u^{-\alpha} \psi .
\end{aligned}
$$


Thus $\int_{\Omega}\langle\nabla u, \nabla \psi\rangle-\int_{\Omega} f(., u) \psi \leq \int_{\Omega} \chi_{\{u>0\}} a u^{-\alpha} \psi$. To prove the existence assertion of the theorem it remains to see that $\chi_{\{u>0\}} a u^{-\alpha} \psi \in L^{1}(\Omega)$, and that

$$
\int_{\Omega}\langle\nabla u, \nabla \psi\rangle-\int_{\Omega} f(., u) \psi \geq \int_{\Omega} \chi_{\{u>0\}} a u^{-\alpha} \psi
$$

for any nonnegative $\psi \in H_{0}^{1}(\Omega) \cap L^{\infty}(\Omega)$. Assume temporarily that $\psi$ satisfies the additional condition $\|\psi\|_{\infty} \leq \frac{1}{2}$, and let $t \in(0,1)$. Note that $u+t \psi \in D_{M}^{\alpha}$. Indeed, by Lemma 2.8 we have $u \leq \mathscr{M}$, and so $0 \leq u+t \psi \leq \mathscr{M}+1 \leq M$. Also $u+t \psi \in H_{0}^{1}(\Omega)$. Thus $u+t \psi \in D_{M}^{\alpha}$ when $0<\alpha<1$.

If $1<\alpha<3$, then $0 \leq a(u+t \psi)^{1-\alpha} \leq a u^{1-\alpha} \in L^{1}(\{a>0\})$, and so $u+t \psi \in D_{M}^{\alpha}$.

If $\alpha=1$, we have $a|\ln (u+t \psi)| \leq a(u+t \psi)$ in $\{a>0\} \cap\{u+t \psi \geq 1\}$, and $a|\ln (u+t \psi)| \leq$ $a|\ln (u)|$ in $\{a>0\} \cap\{u+t \psi<1\}$. Thus $a|\ln (u+t \psi)| \in L^{1}(\{a>0\})$, which implies that $u+t \psi \in D_{M}^{\alpha}$.

To prove (3.7) we consider first the case $\alpha \neq 1$ : Using $J(u) \leq J(u+t \psi)$ we obtain

$$
\begin{aligned}
0 & \leq \frac{1}{t}(J(u+t \psi)-J(u)) \\
& =\int_{\Omega}\langle\nabla u, \nabla \psi\rangle+\frac{t}{2} \int_{\Omega}|\nabla \psi|^{2}-\int_{\{a>0\}} \frac{1}{(1-\alpha) t} a\left((u+t \psi)^{1-\alpha}-u^{1-\alpha}\right) \\
& -\frac{1}{t} \int_{\Omega}(F(., u+t \psi)-F(., u)) .
\end{aligned}
$$

If $1<\alpha<3$ we have $u>0$ a.e. in $\{a>0\}$, and so

$$
\begin{aligned}
& \int_{\{a>0\}} \frac{1}{(1-\alpha) t} a\left((u+t \psi)^{1-\alpha}-u^{1-\alpha}\right) \\
& =\int_{\{a>0\} \cap\{u>0\}} \frac{1}{(1-\alpha) t} a\left((u+t \psi)^{1-\alpha}-u^{1-\alpha}\right) \\
& =\int_{\{a>0\} \cap\{u>0\} \cap\{\psi>0\}} \frac{1}{(1-\alpha) t} a\left((u+t \psi)^{1-\alpha}-u^{1-\alpha}\right) .
\end{aligned}
$$

By the mean value theorem $(u+t \psi)^{1-\alpha}-u^{1-\alpha}=(1-\alpha)\left(u+\sigma_{t}\right)^{-\alpha} \psi$ holds a.e. on $\{u>0\} \cap$ $\{\psi>0\}$, where $\sigma_{t}$ is a measurable function (that depends on $t, u$ and $\psi$ ) such that $0<\sigma_{t}<t \psi$. Thus

$$
\begin{aligned}
& \frac{1}{(1-\alpha) t} \int_{\{a>0\} \cap\{u>0\} \cap\{\psi>0\}} a\left((u+t \psi)^{1-\alpha}-u^{1-\alpha}\right) \\
& =\int_{\{a>0\} \cap\{u>0\} \cap\{\psi>0\}} a\left(u+\sigma_{t}\right)^{-\alpha} \psi .
\end{aligned}
$$


Note that $a\left(u+\sigma_{t}\right)^{-\alpha} \psi \geq 0$ and $\lim _{t \rightarrow 0^{+}} a\left(u+\sigma_{t}\right)^{-\alpha} \psi=a u^{-\alpha} \psi$ hold a.e. on the set where $a>0, u>0$, and $\psi>0$; therefore, from (3.9), (3.10) and Fatou's Lemma, we get

$$
\begin{aligned}
& \underline{\lim }_{t \rightarrow 0^{+}} \int_{\{a>0\}} \frac{1}{(1-\alpha) t} a\left((u+t \psi)^{1-\alpha}-u^{1-\alpha}\right) \\
& \geq \int_{\{a>0\} \cap\{u>0\} \cap\{\psi>0\}} \underline{\lim }_{t \rightarrow 0^{+}} a\left(u+\sigma_{t}\right)^{-\alpha} \psi \\
& =\int_{\{a>0\} \cap\{u>0\} \cap\{\psi>0\}} a u^{-\alpha} \psi=\int_{\Omega} \chi_{\{u>0\}} a u^{-\alpha} \psi .
\end{aligned}
$$

Consider now the case $0<\alpha<1$ : we again apply the mean value theorem to get a measurable function $\sigma_{t}:\{\psi>0\} \rightarrow \mathbb{R}$ (which depends on $t, u$ and $\psi$ ) that satisfies $0<\sigma_{t}<t \psi$, and

$$
\begin{aligned}
& \underline{\lim }_{t \rightarrow 0^{+}} \int_{\{a>0\}} \frac{1}{(1-\alpha) t} a\left((u+t \psi)^{1-\alpha}-u^{1-\alpha}\right) \\
& =\underline{\lim }_{t \rightarrow 0^{+}} \int_{\{a>0\} \cap\{\psi>0\}} \frac{1}{(1-\alpha) t} a\left((u+t \psi)^{1-\alpha}-u^{1-\alpha}\right) \\
& \geq \int_{\{a>0\} \cap\{\psi>0\}} \underline{\lim }_{t \rightarrow 0^{+}}\left(a\left(u+\sigma_{t}\right)^{-\alpha} \psi\right) \\
& =\int_{\{a>0\} \cap\{\psi>0\}} a u^{-\alpha} \psi,
\end{aligned}
$$

where $\left(a u^{-\alpha} \psi\right)(x):=\infty$ if $a(x)>0, \psi(x)>0$, and $u(x)=0$. Thus, for $\alpha \in(0,1) \cup(1,3)$, we have

$$
\underline{\lim }_{t \rightarrow 0^{+}} \frac{1}{(1-\alpha) t} \int_{\{a>0\}} a\left((u+t \psi)^{1-\alpha}-u^{1-\alpha}\right) \geq \int_{\Omega} \chi_{\{u>0\}} a u^{-\alpha} \psi .
$$

Also, by Lemma 2.3 ii), we have

$$
\lim _{t \rightarrow 0^{+}} \frac{1}{t} \int_{\Omega}(F(., u+t \psi)-F(., u))=\int_{\Omega} f(., u) \psi
$$

Now, from (3.8),

$$
\begin{aligned}
& \frac{1}{(1-\alpha) t} \int_{\{a>0\} \cap\{u>0\} \cap\{\psi>0\}} a\left((u+t \psi)^{1-\alpha}-u^{1-\alpha}\right) \\
& \leq \int_{\Omega}\langle\nabla u, \nabla \psi\rangle-\frac{1}{t} \int_{\Omega}(F(., u+t \psi)-F(., u))+\frac{t}{2} \int_{\Omega}|\nabla \psi|^{2}
\end{aligned}
$$

and so, for $1<\alpha<3$, taking $\underline{\lim }_{t \rightarrow 0^{+}}$in (3.14), and using (3.11), (3.12), and (3.13), we get

$$
\int_{\Omega}\langle\nabla u, \nabla \psi\rangle-\int_{\Omega} f(., u) \psi \geq \int_{\Omega} \chi_{\{u>0\}} a u^{-\alpha} \psi \text { if } 1<\alpha<3,
$$


which, in particular, gives $\chi_{\{u>0\}} a u^{-\alpha} \psi \in L^{1}(\Omega)$. Since both sides in (3.15) are linear on $\psi$, the additional assumption $\|\psi\|_{\infty} \leq \frac{1}{2}$ can be removed. Then $u$ is a solution to (1.1) when $1<\alpha<3$; and since $u \in D_{M}^{\alpha}$, it satisfies $u>0$ a.e. in $\{a>0\}$.

Similarly, if $0<\alpha<1$, taking $\underline{\lim }_{t \rightarrow 0^{+}}$in (3.14), and using (3.11), (3.12) and (3.13), we get

$$
\int_{\Omega}\langle\nabla u, \nabla \psi\rangle-\int_{\Omega} f(., u) \psi \geq \int_{\{a>0\} \cap\{\psi>0\}} a u^{-\alpha} \psi \text { if } 0<\alpha<1
$$

for any nonnegative $\psi \in H_{0}^{1}(\Omega) \cap L^{\infty}(\Omega)$. In particular (3.16) gives that $u>0$ a.e. in $\{a>0\} \cap$ $\{\psi>0\}$. From this fact, we conclude (using Remark 2.9 applied with $w=a \chi_{\{u=0\}}$ ) that $u>0$ a.e. in $\{a>0\}$. Then $\int_{\{a>0\} \cap\{\psi>0\}} a u^{-\alpha} \psi=\int_{\Omega} \chi_{\{u>0\}} a u^{-\alpha} \psi$; and so, if $0<\alpha<1$, (3.16) becomes

$$
\int_{\Omega}\langle\nabla u, \nabla \psi\rangle-\int_{\Omega} f(., u) \psi \geq \int_{\Omega} \chi_{\{u>0\}} a u^{-\alpha} \psi
$$

which, in particular, gives $\chi_{\{u>0\}} a u^{-\alpha} \psi \in L^{1}(\Omega)$. Summing up, when $\alpha \neq 1, u>0$ a.e. in $\{a>0\}$; and, for any nonnegative $\psi \in H_{0}^{1}(\Omega) \cap L^{\infty}(\Omega), \chi_{\{u>0\}} a u^{-\alpha} \psi \in L^{1}(\Omega)$, and (3.7) holds.

When $\alpha=1$, the same facts can be proved proceeding, line by line, as in the case $1<\alpha<3$, but with $\frac{1}{1-\alpha} a\left((u+t \psi)^{1-\alpha}-u^{1-\alpha}\right)$ replaced by $a(\ln (u+t \psi)-\ln u)$; and using that, on the set $\{u>0\} \cap\{\psi>0\}$, we have

$$
\ln (u+t \psi)-\ln u=\frac{1}{2}(u+\sigma)^{-1} t \psi
$$

for some measurable function $\sigma$, that depends on $t, u$ and $\psi$, and satisfies $0<\sigma<t \psi$.

Finally, if $f(., 0)>0$ a.e. in $\Omega$, by (3.6) we have, for any nonnegative $\psi \in H_{0}^{1}(\Omega) \cap L^{\infty}(\Omega)$,

$$
\int_{\Omega}\langle\nabla u, \nabla \psi\rangle-\int_{\Omega} \chi_{\{u>0\}} f(., u) \psi \leq \int_{\Omega} \chi_{\{u>0\}} a u^{-\alpha} \psi .
$$

which, jointly with (3.15), implies $\int_{\Omega} f(., u) \psi \leq \int_{\Omega} \chi_{\{u>0\}} f(., u) \psi$; and then $\int_{\{u=0\}} f(., u) \psi \leq$ 0 . Since $f(., 0)>0$ a.e. in $\Omega$, it follows that $\chi_{\{u=0\}} f(., 0) \psi=0$ a.e. in $\Omega$ for any nonnegative $\psi \in C_{c}^{\infty}(\Omega)$. Thus, by Remark 2.9, $\chi_{\{u=0\}} f(., 0)=0$ a.e. in $\Omega$, and then $|\{u=0\}|=0$.

Remark 3.1. If $f \leq 0$, condition $H 4$ ) is automatically fulfilled; indeed, in this case $H 4$ ') holds.

Proof of Theorem 1.3. Since for $\lambda \geq 0, \lambda f$ satisfies the same assumptions fulfilled by $f$, the first assertion of the theorem follows from Theorem 1.2. If, in addition, $\lim _{s \rightarrow \infty} \frac{f(x, s)}{s}=0$ uniformly on $\Omega$, then $-f$ satisfies $H 1)-H 3$ ), H5), and $H 4$ ”); and so, for $\lambda<0$, writing $\lambda f=$ 
$-\lambda(-f)$, the second assertion of the theorem follows from the first one. Finally, if $\lambda \geq 0$ and $f(., 0)>0$ a.e. in $\Omega$, the statement $u_{\lambda}>0$ a.e. in $\Omega$ follows from Theorem 1.2, again.

Proof of Theorem 1.4. Assume that H6) holds. Let $m$ be an integer such that $m \geq \max \{2,1+\alpha\}$, and let $k \in \mathbb{R}$ satisfy $k>\max \left\{0, \operatorname{ess}_{\sup } \operatorname{se}_{\Omega \times(\infty)} \frac{f(x, s)}{s}\right\}$. Thus $\frac{\lambda f(., s)}{s} \leq \lambda k$ and, since $\lambda_{1}(\lambda k \mathbf{1})=\frac{\lambda_{1}(\mathbf{1})}{\lambda k}>m$ for $0<\lambda<\frac{\lambda_{1}(\mathbf{1})}{m k}$, Theorem 1.2 gives, for such $\lambda$, the sought weak solution of (1.2). Note also that, if $\lambda=0,(1.2)$ reduces to $-\Delta u=\chi_{\{u>0\}} a u^{-\alpha}$ in $\Omega, u \geq 0$ in $\Omega, u=0$ on $\partial \Omega$; and this problem has a positive weak solution $u \in H_{0}^{1}(\Omega) \cap L^{\infty}(\Omega)$ (see [10]). Then the lemma holds with $\lambda^{*}:=\frac{\lambda_{1}(\mathbf{1})}{m k}$.

Assume now that H7) holds. Let $V:=\left\{k \in(0, \infty): f(., k) \in L^{\infty}(\Omega)\right\}$. Since $f \in L^{\infty}(\Omega \times(0, \sigma))$ for any $\sigma>0$, we have that $\mathbb{R} \backslash V$ has zero Lebesgue's measure. For $k \in V$ to be chosen latter, let $f_{k}: \Omega \times[0, \infty)$ be defined by $f_{k}(., s):=f(., s)$ if $0 \leq s \leq k$, and by $f_{k}(., s):=f(., k)$ otherwise. Let $\lambda>0$; clearly $\lambda f_{k}$ satisfies the conditions H2),H3) and H5). Since $f(., k) \in L^{\infty}(\Omega)$, we have $\overline{\lim }_{s \rightarrow \infty} \frac{\lambda f_{k}(., s)}{s}=0$ uniformly on $\Omega$, and so $\lambda f_{k}$ satisfies also $H 4$ ”). Let $u \in H_{0}^{1}(\Omega) \cap L^{\infty}(\Omega)$ be the solution to the problem:

$$
\left\{\begin{array}{l}
-\Delta u=\chi_{\{u>0\}} a u^{-\alpha}+\lambda f_{k}(x, u) \text { in } \Omega, \\
u=0 \text { on } \partial \Omega, \\
u \geq 0 \text { in } \Omega, u \neq \equiv \text { in } \Omega,
\end{array}\right.
$$

provided by Theorem 1.2. Thus $u$ satisfies $\chi_{\{u>0\}} a u^{-\alpha} \not \equiv 0$. Let $m$ be an integer such that $m \geq \max \{2,1+\alpha\}$, let $\lambda_{1}$ be the first eigenvalue for $-\Delta$ on $\Omega$ with homogeneous Dirichlet condition, let $\eta \in(0,1)$, and let $\varepsilon:=\eta \frac{\lambda_{1}}{\lambda m}$. Take $\Lambda \in(0, \infty)$, and define

$$
s_{0}:=\max \left\{\frac{m \Lambda\|f(., k)\|_{\infty}}{\eta \lambda_{1}}, k\right\}
$$

Thus, for $s>s_{0}$ and $0 \leq \lambda<\Lambda$,

$$
\frac{\lambda\left|f_{k}(., s)\right|}{s} \leq \frac{\Lambda|f(., k)|}{s_{0}} \leq \frac{\eta \lambda_{1}}{m}<\frac{\lambda_{1}}{m} \text { a.e. in } \Omega \text {. }
$$

From the proof of Theorem 1.2 we know that, for $M$ positive and large enough, $u$ is a minimizer for $J_{\lambda f_{k}}$ on $D_{M}^{\alpha}$; and so, by Lemma 2.6, we have, in the weak sense stated there (i.e., for test 
functions in $\left.H_{0}^{1}(\Omega) \cap L^{\infty}(\Omega)\right)$,

$$
\begin{aligned}
-\Delta\left(u^{m}\right) & \leq m a\left(u^{m}\right)^{\frac{m-1-\alpha}{m}}+m u^{m-1} \lambda f_{k}(., u) \\
& \leq m a\left(u^{m}\right)^{\frac{m-1-\alpha}{m}}+\lambda m A s_{0}^{m-1}+m u^{m} \chi_{u>s_{0}} \frac{\lambda\left|f_{k}(., u)\right|}{u} \\
& \leq m a u^{m-1-\alpha}+\lambda m A s_{0}^{m-1}+\eta \lambda_{1} u^{m}
\end{aligned}
$$

for $0 \leq \lambda<\Lambda$ and with $A:=1+\left\|f_{\mid \Omega \times\left(0, s_{0}\right)}\right\|_{L^{\infty}\left(\Omega \times\left(0, s_{0}\right)\right)}<\infty$. As $m a\left(u^{m}\right)^{\frac{m-1-\alpha}{m}}+\lambda m A s_{0}^{m-1}+$ $\eta \lambda_{1} u^{m} \in L^{2}(\Omega)$, Remark 2.7 says

$$
-\Delta\left(u^{m}\right) \leq m a\left(u^{m}\right)^{\frac{m-1-\alpha}{m}}+\lambda m A s_{0}^{m-1}+\eta \lambda_{1} u^{m}
$$

in the usual $H_{0}^{1}(\Omega)$ weak sense ( i.e., for arbitrary test functions in $H_{0}^{1}(\Omega)$ ). Now, $\eta \lambda_{1}<\lambda_{1}$, and so $\left(-\Delta-\eta \lambda_{1}\right)^{-1}: L^{\infty}(\Omega) \rightarrow L^{\infty}(\Omega)$ is a well defined, bounded, and positive operator; let $c:=\left\|\left(-\Delta-\eta \lambda_{1}\right)^{-1}\right\|_{L^{\infty}(\Omega), L^{\infty}(\Omega)}$. Then, since $u$ is nonnegative, from (3.19) we get

$$
\|u\|_{\infty}^{m} \leq c\left(m\|a\|_{\infty}\|u\|_{\infty}^{m-1-\alpha}+\lambda m A s_{0}^{m-1}\right) .
$$

Then, either $\|u\|_{\infty}^{m} \leq 2 c m\|a\|_{\infty}\|u\|_{\infty}^{m-1-\alpha}$, or $\|u\|_{\infty}^{m} \leq 2 c \lambda m A s_{0}^{m-1}$. Now we choose $k \in V$ such that $k>\left(2 c m\|a\|_{\infty}\right)^{\frac{1}{1+\alpha}}$. If $\|u\|_{\infty}^{m} \leq 2 c m\|a\|_{\infty}\|u\|_{\infty}^{m-1-\alpha}$, then $\|u\|_{\infty} \leq k$; therefore $f_{k}(, . u)=$ $f(, . u)$, and so $u$ is a solution to (1.2). If $\|u\|_{\infty}^{m} \leq 2 c \lambda m A s_{0}^{m-1}$, then $\|u\|_{\infty} \leq \lambda^{\frac{1}{m}}\left(2 c m A s_{0}^{m-1}\right)^{\frac{1}{m}}$;

and so, if $\lambda \in\left[0, \lambda^{*}\right)$ with $\lambda^{*}:=\min \left\{\Lambda, \frac{k^{m}}{2 c m A s_{0}^{m-1}}\right\}$, we have $u \leq k$, which implies that $u$ solves (1.2). Finally, the conclusion $u_{\lambda}>0$ a.e. in $\{a>0\}$ follows from Theorem 1.2 used with $f_{k}$ instead of $f$.

Remark 3.2. Assume H1)-H3), H5) and $f \leq 0$. Then (1.2) has a weak solution (in the sense of Definition 1.1) for all $\lambda \geq 0$. Indeed, this follows from Theorem 1.2 applied with $\lambda f$ instead of $f$.

\section{REFERENCES}

[1] B. Bougherara, J. Giacomoni, Existence of mild solutions for a singular parabolic equation and stabilization, Adv. Nonlinear Anal. 4 (2015), 123-134.

[2] H. Brezis, Functional Analysis, Sobolev Spaces and Partial Differential Equations, Springer, 2011.

[3] B. Bougherara, J. Giacomoni, J. Hernández, Existence and regularity of weak solutions for singular elliptic problems, 2014 Madrid Conference on Applied Mathematics in honor of Alfonso Casal, Electron. J. Diff. Equ. Conference 22 (2015), 19-30. 
[4] A. Callegari, A. Nashman, A nonlinear singular boundary-value problem in the theory of pseudoplastic fluids, SIAM J. Appl. Math. 38 (1980), 275-281.

[5] M.M. Coclite, G. Palmieri, On a singular nonlinear Dirichlet problem, Comm. Part. Differ. Equat. 14 (10), (1989), 1315-1327.

[6] D. S. Cohen, H. B. Keller, Some positive problems suggested by nonlinear heat generators, J. Math. Mech. 16 (1967), 1361-1376.

[7] M. G. Crandall, P. H. Rabinowitz, L. Tartar, On a Dirichlet problem with a singular nonlinearity, Comm. Part. Differ. Equations 2 (1977), 193-222.

[8] F. Cîrstea, M. Ghergu, V. Rădulescu, Combined effects of asymptotically linear and singular nonlinearities in bifurcation problems of Lane-Emden-Fowler type, J. Math. Pures Appl. 84 (2005), 493-508.

[9] J. Dávila, M. Montenegro, Positive versus free boundary solutions to a singular elliptic equation, J. Anal. Math. 90 (2003), 303-335.

[10] M. A. del Pino, A global estimate for the gradient in a singular elliptic boundary value problem, Proc. R. Soc. Edinburgh Sect. A 122 (1992), 341-352.

[11] J. I. Díaz, J. Hernández, Positive and free boundary solutions to singular nonlinear elliptic problems with absorption; An overview and open problems, Variational and Topological Methods: Theory, Applications, Numerical Simulations, and Open Problems (2012). Electron. J. Diff. Equ., Conference 21 (2014), 31-44.

[12] J. Díaz, M. Morel, L. Oswald, An elliptic equation with singular nonlinearity, Comm. Part. Diff. Eq. 12 (1987), 1333-1344.

[13] D. G. De Figueiredo, Positive solutions of semilinear elliptic equations, Lect. Notes Math. Springer, 957 (1982), 34-87.

[14] L. Dupaigne, M. Ghergu, V. Rădulescu, Lane-Emden-Fowler equations with convection and singular potential, J. Math. Pures Appl. 87 (2007), 563-581.

[15] W. Fulks, J. S. Maybee, A singular nonlinear equation, Osaka Math. J. 12 (1960), 1-19.

[16] M. Ghergu, V. Liskevich, Z. Sobol, Singular solutions for second-order non-divergence type elliptic inequalities in punctured balls, J. Anal. Math. 123 (2014), 251-279.

[17] M. Ghergu, V. D. Rădulescu, Singular Elliptic Problems: Bifurcation and Asymptotic Analysis, Oxford Lecture Series in Mathematics and Its Applications, Oxford University Press, No 37, 2008.

[18] M. Ghergu, V. D. Rădulescu, Multi-parameter bifurcation and asymptotics for the singular Lane-EmdenFowler equation with a convection term, Proc. Royal Soc. Edinburgh, Sect. A 135 (2005), 61-84.

[19] T. Godoy, A. Guerin, Nonnegative solutions of a singular elliptic problem, Electron. J. Diff. Equ. 2016 (2016), Article ID 191.

[20] V. V. Kurta, A Liouville comparison principle for solutions of quasilinear singular parabolic inequalities, Adv. Nonlinear Anal. 4 (2015), 1-11.

[21] A. C. Lazer, P. J. McKenna, On a singular nonlinear elliptic boundary value problem, Proc. Amer. Math. Soc. 111 (1991), 721-730. 
[22] B. B. Manna, P. C. Srikanth, On the solutions of a singular elliptic equation concentrating on a circle, Adv. Nonlinear Anal. 3 (2014), 141-155.

[23] V. D. Rădulescu, Singular phenomena in nonlinear elliptic problems, From blow-up boundary solutions to equations with singular nonlinearities, in Handbook of Differential Equations: Stationary Partial Differential Equations, Vol. 4 (M. Chipot, Editor), North-Holland Elsevier Science, Amsterdam, 2007, pp. 483-591.

[24] J. Shi, M. Yao, On a singular nonlinear semilinear elliptic problem, Proc. R. Soc. Edinburgh, Sect A 128 (1998), 1389-1401. 